\title{
Determination of Transmembrane Water Fluxes in Neurons Elicited by Glutamate Ionotropic Receptors and by the Cotransporters KCC2 and NKCC1: A Digital Holographic Microscopy Study
}

\author{
Pascal Jourdain, ${ }^{1}$ Nicolas Pavillon, ${ }^{2}$ Corinne Moratal, ${ }^{1}$ Daniel Boss, ${ }^{1}$ Benjamin Rappaz,,${ }^{1}$ Christian Depeursinge, ${ }^{2}$ \\ Pierre Marquet, ${ }^{1,3 *}$ and Pierre J. Magistretti ${ }^{1,3 *}$ \\ ${ }^{1}$ Brain and Mind Institute, and ${ }^{2}$ Advanced Photonics Laboratory, Microvision and Microdiagnostic Group, École Polytechnique Fédérale de Lausanne, 1015 \\ Lausanne, Switzerland, and ${ }^{3}$ Department of Psychiatry-University Hospital, Centre de Neurosciences Psychiatriques, 1008 Prilly-Lausanne, Switzerland
}

\begin{abstract}
Digital holographic microscopy (DHM) is a noninvasive optical imaging technique that provides quantitative phase images of living cells. In a recent study, we showed that the quantitative monitoring of the phase signal by DHM was a simple label-free method to study the effects of glutamate on neuronal optical responses (Pavillon et al., 2010). Here, we refine these observations and show that glutamate produces the following three distinct optical responses in mouse primary cortical neurons in culture, predominantly mediated by NMDA receptors: biphasic, reversible decrease (RD) and irreversible decrease (ID) responses. The shape and amplitude of the optical signal were not associated with a particular cellular phenotype but reflected the physiopathological status of neurons linked to the degree of NMDA activity. Thus, the biphasic, RD, and ID responses indicated, respectively, a low-level, a high-level, and an "excitotoxic" level of NMDA activation. Moreover, furosemide and bumetanide, two inhibitors of sodium-coupled and/or potassium-coupled chloride movement strongly modified the phase shift, suggesting an involvement of two neuronal cotransporters, NKCC1 (Na-K-Cl) and KCC2 (K-Cl) in the genesis of the optical signal. This observation is of particular interest since it shows that DHM is the first imaging technique able to monitor dynamically and in situ the activity of these cotransporters during physiological and/or pathological neuronal conditions.
\end{abstract}

\section{Introduction}

The passive diffusion or active transport of water through the plasma membrane is associated with several cellular processes. As far as the nervous system is concerned, the neuronal activity resulting in glutamate release has been shown to result in cell swelling linked to water diffusion (Andrew and MacVicar, 1994; Schwartzkroin et al., 1998). The actions of glutamate are mediated by both ionotropic and metabotropic receptors (Meldrum, 2000). However, it is well established that an excessive NMDA receptor activation induces intense neuronal depolarization and, as a consequence, an excessive $\mathrm{Na}^{+}, \mathrm{Ca}^{2+}, \mathrm{Cl}^{-}$, and water inflow that causes rapid cell swelling, osmolysis, and other $\mathrm{Ca}^{2+}$ -

\footnotetext{
Received Jan. 18, 2011; revised May 23, 2011; accepted June 22, 2011.

Author contributions: P.M. and P.J.M. designed research;P.J., N.P., C.M., D.B., B.R., and C.D. performed research; P.J., N.P., and D.B. analyzed data; P.J., P.M., and P.J.M. wrote the paper.

This research has been funded by Commission for Technology and Innovation project no. 9389.1 and by the Swiss National Science Foundation (SNSF) Grants no. CR3213_132993 and no. 31003AB_131087. We thank Lyncée Tech SA (www.lynceetec.com) for technological assistance and Aurelie Calame for neuronal culture preparation.

*P.M. and P.J.M. are co-last authors.

C.D., PJ.M., and P.M. are founders of Lyncée Tec

Correspondence should be addressed to either Prof. Pierre J. Magistretti or Pascal Jourdain, Brain and Mind Institute, Ecole Polytechnique Fédérale de Lausanne, 1015 Lausanne, Switzerland. E-mail: pierre.magistretti@epfl. ch or pascal.jourdain@epfl.ch.

DOI:10.1523/JNEUROSCI.0286-11.2011

Copyright $\odot 2011$ the authors $\quad 0270-6474 / 11 / 3111846-09 \$ 15.00 / 0$
}

dependent processes damageable to neurons (Rothman, 1985; Olney et al., 1986).

In addition to receptor activation, other important regulatory elements of neuronal excitability and cellular volume are the two cotransporters, NKCC1 and KCC2, notably involved in $\mathrm{Cl}^{-}$ equilibrium (MacAulay et al., 2004). Activation of the KCC2 leads to the net loss of $\mathrm{K}^{+}$and $\mathrm{Cl}^{-}$accompanied by water (MacAulay et al., 2004). In contrast, activation of NKCC1 triggers a net inflow of $\mathrm{K}^{+}, \mathrm{Na}^{+}$, and $\mathrm{Cl}^{-}$with a stoichiometry of 1,1 , and 2, respectively. This inflow of ions is associated with an inflow of water (Russell, 2000).

While water transport has been used as an indirect marker of neuronal activation by certain imaging techniques such as transmittance imaging (Grinvald et al., 1986; Andrew and MacVicar, 1994; Andrew et al., 1996) or even functional MRI (Le Bihan, 2007), at the cellular level a recently developed type of noninvasive label-free cell imaging, digital holographic microscopy (DHM), allows the monitoring of absolute cell volume as well as the associated transmembrane flow of water, with the additional advantage of being quantitative (Rappaz et al., 2005; Pavillon et al., 2010). Briefly, DHM is an interferometric imaging technique that measures quantitatively the phase shift induced on the transmitted wave by the observed specimen. Consequently, the phase signal provides information about cell morphology (and volume) and the intracellular refractive index whose value is related to the amount of nonaqueous material (essentially protein content) 
present in the cell (Barer, 1953). However, recent studies have shown that phase signal changes mainly reflect intracellular refractive index variations (Rappaz et al., 2005; Pavillon et al., 2010). Consequently, within this framework, the phase signal will be predominantly sensitive to any mechanism modifying the concentration of intracellular compounds, including transmembrane water movements, which accompany various ionic fluxes. Concretely, an entry of water will dilute the intracellular content, producing a decrease in the phase signal. In contrast, an outflow of water will concentrate the intracellular content, resulting, in this case, in an increase in the phase signal.

In this study, we have taken advantage of the possibility of DHM to monitor transmembrane water fluxes as detected by the phase signal, to assess the activity of NKCC1 and KCC2 in response to depolarizations induced by glutamate on primary neurons in culture. The results obtained show indeed that the activity of NKCC1 and KCC2 can be studied noninvasively by simple analysis of the phase optical signal detected by DHM.

\section{Materials and Methods}

Culture preparation. Primary cultures of cortical neurons were prepared from E17 OF1 mice embryos of either sex. Briefly, embryos were decapitated, and brains removed and placed in PBS-glucose. Cortices were removed under a dissecting microscope and collected in a small Petri dish in PBS-glucose. A single-cell suspension was obtained by gentle trituration with a fire-polished Pasteur pipette in Neurobasal medium supplemented with B27 and GlutaMAX (Invitrogen). Cells were plated at an average density of $15,000 \mathrm{cells} / \mathrm{cm}^{2}$ in supplemented Neurobasal medium on polyornithine-coated glass coverslips $(20 \mathrm{~mm} \varnothing)$. After 3-4 h, coverslips were transferred to dishes containing glial cell monolayers in supplemented Neurobasal medium. Neurons were maintained at $37^{\circ} \mathrm{C}$ in a humidified atmosphere of $95 \%$ air $/ 5 \% \mathrm{CO}_{2}$ and were used after $21-35$ $\mathrm{d}$ in vitro (DIV).

Electrophysiology recordings. Primary cultures were perfused in an artificial CSF (ACSF) containing the following (in $\mathrm{mM}$ ): $\mathrm{NaCl} \mathrm{150,} \mathrm{KCl} 3$, D-glucose 10, HEPES 10, $\mathrm{CaCl}_{2} 3$, and $\mathrm{mm} \mathrm{MgCl}_{2}$. 2, $\mathrm{pH} 7.4$ (room temperature). For some experiments, CNQX (40 $\mu \mathrm{M}$, Tocris Bioscience), MK801 (40 $\mu \mathrm{M}$, Sigma), TTX (1 $\mu \mathrm{M}$, Sigma), furosemide (100 $\mu \mathrm{M}$, Sigma), bumetanide ( $20 \mu \mathrm{M}$, Sigma), picrotoxin (30 $\mu \mathrm{M}$, Tocris Bioscience), or GABA ( $3 \mu \mathrm{M}$, Tocris Bioscience) was added to the ACSF. For glycine $+\mathrm{Mg}^{2+}$-free medium, Glycine (10 $\mu \mathrm{M}$, Sigma) is added to ACSF and $\mathrm{MgCl}_{2}$ is substituted with $\mathrm{CaCl}_{2}$ in equimolar manner. Glutamate (30 $\mu \mathrm{M}$, Tocris Bioscience) were dissolved in ACSF and applied by bath perfusion (for $30 \mathrm{~s}$ ). Whole-cell recordings were made, and signals were amplified by using Multiclamp 700B amplifiers (Molecular Devices) and were digitized by means of an ITC-1600 interface (Instrutech) to a PC computer running Igor Pro (Wavemetrics). All currents (sampling frequency, 10 $\mathrm{kHz}$ ) were low-pass filtered $(2 \mathrm{kHz})$. They were recorded with pipettes containing the following (in $\mathrm{mm}$ ): 110 potassium gluconate, $10 \mathrm{KCl}, 4 \mathrm{ATP}-\mathrm{Mg}$, 10 phosphocreatine, 0.3 GTP, $10 \mathrm{~N}$-2-hydroxyethylpiperazine- $N^{\prime}-2$ ethanesulfonic acid, pH 7.3 (310 mOsm adjusted with sucrose), and $0.5 \%$ biocytin (Sigma). The pipettes were pulled with a DMZ universal puller.

Imaging. The basic design of our imaging system has been described by Marquet et al. (2005) and Rappaz et al. (2005). Briefly, holograms are acquired with a DHMT 1000 (Lyncée Tech SA, Parc Scientifique-École Polytechnique Fédérale de Lausanne). A laser diode is used as a coherent light source $(\lambda=683 \mathrm{~nm})$, which is divided by a beam splitter into a reference wave and an object wave. The object wave diffracted by the specimen is collected by a microscope objective and interferes with a reference beam to produce the hologram recorded by the CCD camera. The frequency of hologram acquisition is $0.2 \mathrm{~Hz}$. Reconstruction of the original image from the hologram is numerically achieved by a computer. The reconstruction algorithm provides simultaneous amplitude and quantitative phase images of the cells (Koala Software). An extensive quality control of the DHM technique has been published by Rappaz et al. (2008).

For all experiments, glutamate was added after a minimum of 1 min of stable baseline recording for the phase signals. Note that in certain cases, notably cell-death experiments, optical recordings lasted up to $4 \mathrm{~h}$. Note that cells that had not developed a detectable optical response after application of glutamate were excluded. They represented $\sim 10-15 \%$ of total cells.

Immunocytochemistry. For some experiments, the intrapipette medium contained $0.1 \%$ of biocytin (Sigma). By simple intracellular dialysis during a whole-cell patch-clamp recording, individual neurons were filled with biocytin. The cultures were then fixed in $4 \%$ paraformaldehyde (15 min at room temperature). After thorough rinsing in PBS, the cultures underwent immunocytochemical staining. For this, cultures were treated first with PBS containing $0.25 \%$ Triton X-100 (10 min) to permeabilize plasma membranes and after with PBS containing $0.25 \%$ bovine serum albumin (BSA; $30 \mathrm{~min}$ ) to reduce nonspecific binding of antibodies. They were then incubated overnight at $4^{\circ} \mathrm{C}$ with a rabbit polyclonal antibody anti-glutamic acid decarboxylase (GAD) diluted 1:1000 (Millipore Bioscience Research Reagents). After rinsing with PBS, cultures were treated with a mixture of streptavidin conjugated with Texas Red (1:400; Invitrogen) to reveal biocytin and goat anti-rabbit Igs Alexa Fluor 488 (1:200; Invitrogen) to visualize the anti-GAD. All antibodies were dissolved in PBS containing $0.25 \%$ BSA. The cultures were mounted with DABCO (Sigma) and examined under epifluorescence microscopy (DMI 4000, Leica) with appropriate filters.

Cell viability assessment. At the end of experiment, cell viability was assessed with propidium iodide (Sigma-Aldrich) labeling, by adding the reagent at a concentration of $7.5 \mu \mathrm{M}$ in the extracellular medium ACSF. After $30 \mathrm{~min}$ to $1 \mathrm{~h}$ of charging, cells were measured in epifluorescence microscopy with appropriate filters to detect labeled nuclei, which indicated a loss in membrane integrity and subsequent cell death.

Off-line analysis. The optical and electrophysiological recordings were analyzed by using MATLAB 7.6 (Mathworks Software) and MiniAnalysis Program 6.0.7 (Synaptosoft), respectively. The template chosen for each current event consisted of a 5 ms baseline, complete rise time $\left(\tau_{\text {rise }}\right)$ and $90 \%$ of decay time $\left(\tau_{\text {decay }}\right)$. Only single-peak events were accepted during subsequent visual control. One hundred or more events were averaged in a given experiment. Events were identified as sEPSCs by setting the event detection threshold at $5 \mathrm{pA}$, and checking that events had (1) rise times faster than decay times, (2) rise times $>0.4 \mathrm{~ms}$, and (3) decay times $>1.5 \mathrm{~ms}$. The significance of the shift in cumulative probability distributions of $\tau_{\text {rise }}$ and $\tau_{\text {decay }}$ was assessed by pooling together the data of all the experiments for each condition and using the nonparametric Kolmogorov-Smirnov (KS) two-sample test. For all graphs, curves and statistics we used ORIGIN 8.5 (Microcal Software). Glutamate concentration-response (or time application-response) profiles were fitted to the following logistic equation: $\varphi / \varphi_{\max }=1 /\left[1+\left(\mathrm{EC}_{50} /[\text { glutamate }]\right)^{n}\right]$, where $\varphi$ and $\varphi_{\max }$ represented the normalized glutamate-induced phase shift at a given concentration and the maximum phase shift induced by a saturating glutamate concentration before cell death, $\mathrm{EC}_{50}$ was the halfmaximal $\mathrm{EC}_{50}$, and $n$ was the slope factor. Moreover, the $\mathrm{IC}_{50}$ value for glutamate antagonists and cotransporter blockers were calculated according to the logistic equation: $\varphi=\varphi_{\max } /\left[1+\left([\text { drugs }] / \mathrm{IC}_{50}\right)^{s}\right]$, where $s$ is the Hill slope.

All data are presented as means \pm SEM. Student's $t$ test (paired or unpaired) to determine statistical significance $(p<0.05)$.

\section{Results}

Neurons in culture for 21-35 d were studied by DHM. We distinguished two morphological types of neurons: first, pyramidalshaped neurons with large- and small-diameter dendrites emerging from cell bodies; second, bipolar (or multipolar) neurons corresponding to nonpyramidal interneurons (Fig. 1A).

\section{Glutamate triggered three main types of phase shifts recognizable by their shape and amplitude: biphasic, reversible decrease, and irreversible decrease}

An application of glutamate $(30 \mu \mathrm{M}, 30 \mathrm{~s})$ triggered different types of optical signals in primary cultured neurons (Fig. 1A). These signals can be grouped into the following three main cate- 
gories: (1) a biphasic response (Fig. $1 B, C$ ) characterized by an increase in the phase signal $\left(4.78 \pm 0.58^{\circ}\right)$ (this increase may in certain cases, be preceded by a small initial decrease of the optical signal; $-1.18 \pm$ $\left.1.12^{\circ}\right)$, and this response has been recorded in $54 \%$ of studied cells ( $n=137$ of 252 cells); (2) a reversible decrease (RD) (Fig. $1 B, C$ ) for which the phase signal strongly but transiently decreased $\left(-6.99 \pm 1.34^{\circ}\right)$ (this response has been recorded in $32 \%$ of studied cells; $n=80$ of 252 cells); and (3) an irreversible decrease (ID) (Fig. $1 B, C$ ) of the optical response where the phase signal strongly decreased $(-25.4 \pm 3.79)$ (this response represented only $14 \%$ of recorded cells; $n=35$ of 252 cells).

It was important to note that similar applications of glutamate $(30 \mu \mathrm{M}, 30 \mathrm{~s})$ performed on very young neuronal cultures (8-15 DIV) had little or no effect on the phase signal compared with older cultures (Fig. $1 B$ ), which is consistent with the fact that the expression of glutamate receptors is considerably greater in older than in young cultures (Brewer et al., 2007).

Biphasic and $\mathrm{RD}$ responses are preferentially associated with activation of NMDA receptors

We have determined the type of ionotropic glutamate receptors involved in genesis of biphasic and RD signals. Thus, application of DNQX $(40 \mu \mathrm{M})$, a specific antagonist of non-NMDA receptors, significantly blocked both biphasic $(p<0.005, n=9)$ and $\mathrm{RD}(p<$ $0.05, n=13$ ) responses produced by glutamate (Fig. $2 A$ ), with an $\mathrm{IC}_{50}$ of 120 and $110 \mathrm{~nm}$, respectively, for biphasic $(n=7)$ and RD responses $(n=7)$ (Fig. 3). However, with an environment strongly promoting the NMDA receptor activation $\left(\mathrm{Mg}^{2}{ }^{+}\right.$-free and glycine solution), glutamate triggered optical responses such as biphasic or RD, even in the presence of DNQX (Fig. 2B), suggesting an essential role of NMDA receptors in genesis of optical responses.

In the presence of a specific antagonist of NMDA receptor, MK801 $(40 \mu \mathrm{M})$, the responses to glutamate application were considerably decreased, with only a residual phase response still detectable (Fig. 2 A). This residual MK801-resistant component, which is called a "biphasic-like" signal (its shape is close to the normal biphasic signal but with a weaker amplitude), was not modified with an environment strongly promoting the NMDA receptor activation $\left(\mathrm{Mg}^{2+}\right.$-free and glycine solution) (Fig. $\left.2 \mathrm{~B}\right)$, confirming that the NMDA component has been blocked by MK801 but disappeared in the presence of the non-NMDA receptor antagonist DNQX (40 $\mu \mathrm{M}$; data not shown), suggesting that the biphasic-like signal is a small non-NMDA component of the optical response (biphasic and $\mathrm{RD}$ ) triggered by glutamate. Note that the $\mathrm{IC}_{50}$ values of MK801 were $170 \mathrm{~nm}$ for biphasic response $(n=7)$ and $760 \mathrm{~nm}$ for $\mathrm{RD}$ response $(n=9)$ (Fig. $3 B)$, indicating a stronger involvement of NMDA receptors in RD responses.

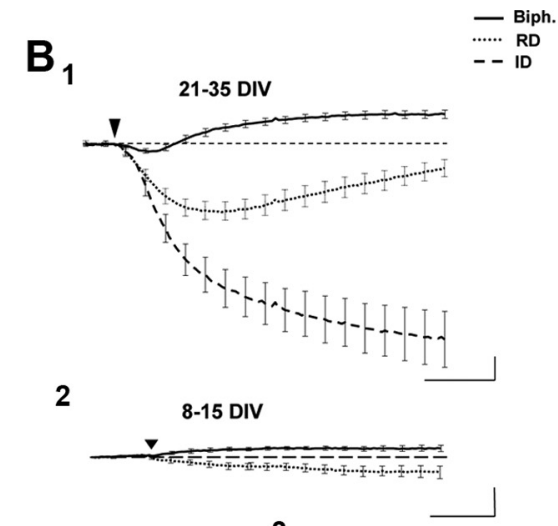

2

3

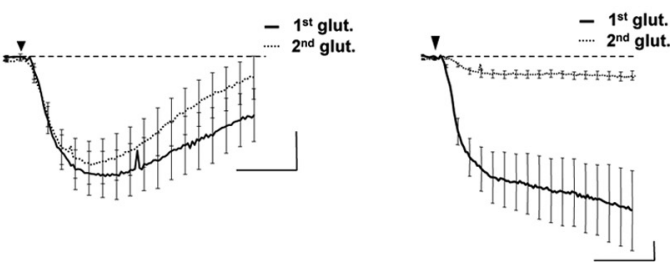

Figure 1. Application of glutamate triggers three main types of phase shifts. $\boldsymbol{A}$, Left, Representative phase image of three (he cortical neurons visualized in DHM. Ovals in the middle of the cells corresponded to the region of interest for the phase (he categories defined in B1. C, Two consecutive averaged biphasic (C1; $n=16), \operatorname{RD}(\mathbf{C} ; n=14)$, and ID (C,$n=$ there was no significant difference between control (first glut.) and the delayed (second glut.) optical response. The delay between two applications was a minimum of $30 \mathrm{~min}$. Calibration: 2 min and $5^{\circ}$.

Finally, when blocking spontaneous synaptic transmission with TTX $(1 \mu \mathrm{M})$, glutamate triggered a biphasic-like response (Fig. 2 A) blocked by DNQX but not by MK801 (data not shown), thus validating the non-NMDA nature of this optical signal. However, when TTX was added in an $\mathrm{Mg}^{2}{ }^{+}$-free and glycine extracellular solution, we retrieved biphasic and/or RD responses triggered by glutamate (Fig. $2 \mathrm{~B}$ ), therefore confirming a strong contribution of the NMDA receptor in the genesis of optical signal, with the non-NMDA receptors playing a role in triggering.

ID responses are the optical signature of cell death associated to NMDA receptor-dependent excitotoxicity

In a previous article, Pavillon et al. (2010) have suggested that the ID response could be associated with an excitotoxic process. Our study confirmed that an ID response would be the optical signature of cell death. First, contrary to the two other optical responses, the ID response is not reproducible since a second application of glutamate could not trigger a phase shift (Fig. 1C), suggesting that this optical signal is associated with a terminal cellular process. Over a longer period of recording, after the rapid strong decrease of the phase-shift signal described in Figure 1, this optical signal reached a new steady-state without returning to the initial value, even $4 \mathrm{~h}$ after the application of glutamate $(\Delta \varphi=$ $-48 \pm 13^{\circ} ; n=6$ ) (Fig. $4 A$ ). Second, like biphasic and RD responses, the ID signal could be obtained when the environment strongly promoting the NMDA receptor activation $\left(\mathrm{Mg}^{2}{ }^{+}\right.$-free and glycine in extracellular solution), even when the non-NMDA receptor was blocked with DNQX (Fig. $4 B$ ). Conversely, the ID response was never recorded in the presence of MK801 (Fig. 2 B), suggesting a strong involvement of NMDA receptor in the genesis 

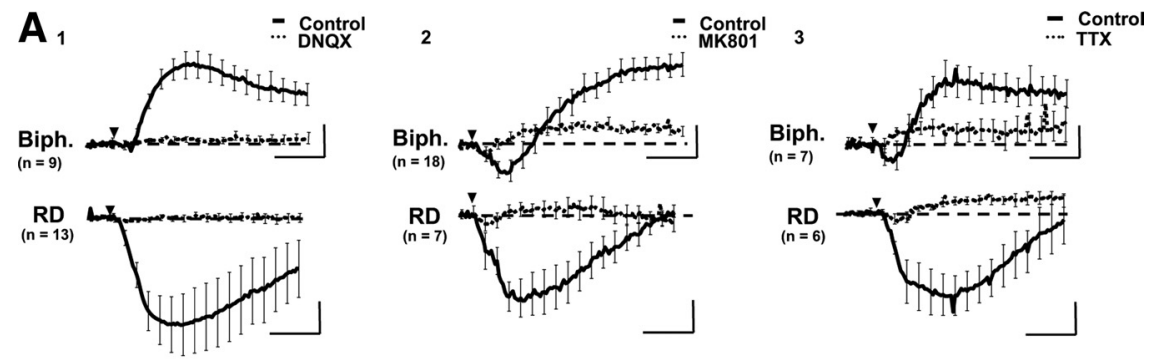

$B_{1}$

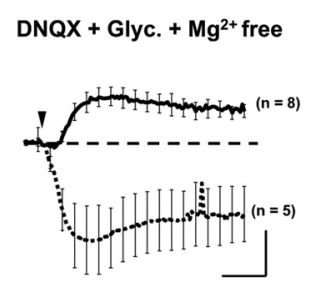

2

MK801 + Glyc. $+\mathrm{Mg}^{2+}$ free

3

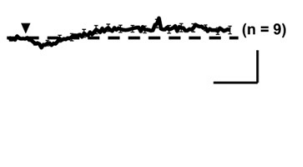

been described as a persistent depolarizing current associated with the pathological process triggered by activation of NMDA receptors leading to cell death (Limbrick et al., 2001). Finally, all cells displaying an ID response and treated with propidium iodide, a marker of cell death (Nicoletti et al., 1991; Lecoeur, 2002), were labeled ( $n=8)$ (Fig. $4 D$ ). Thus, together these results strongly suggest that the ID response is an early optical signature of the subsequent cell death, here associated with the (excitotoxic) activity of NMDA receptors, known to be involved in pathological process (Mody and MacDonald, 1995; Arundine and Tymianski, 2004).

The optical biphasic and $\mathrm{RD}$ responses reflect the excitability status of neurons The previous set of data indicated an involvement of NMDA receptors in the genesis of biphasic, RD, and ID optical responses. While it seems clear that the ID response corresponds to an "excitotoxic" activation of NMDA receptors, the question about the degree of involvement of the NMDA receptors for the other two types of optical signals remains open.

Thus, the concentration-response curve to glutamate ( $300 \mathrm{~nm}$ to $3 \mathrm{~mm}$ ) reveals $\mathrm{EC}_{50}$ values for glutamate of 45 and 15 $\mu \mathrm{M}$, respectively, for biphasic and $\mathrm{RD}$ responses (Fig. $5 A$ ), suggesting that the type of the optical response is related to the sensitivity of neurons to glutamate. This result was consistent with the calculated $\mathrm{IC}_{50}$ values for MK801, showing a marked difference between biphasic (170 nM) and RD response (760 nM) (Fig. 3B). Note that for the ID response, the $\mathrm{EC}_{50}$ for glutamate is $<3 \mu \mathrm{M}$ (data not shown).

To confirm this hypothesis, we recorded the activity of several neurons previously categorized according to their optical responses. Thus, patched cells previously showing an $\mathrm{RD}$ response (RD cells; $n=20$ ) displayed a more depolarized resting potential than the neurons developing a biphasic response (biphasic cells; $n=12 ;-46$ vs $-57 \mathrm{mV}$, respectively; $p<0.005$ ), meaning that RD cells were in a state of greater excitability. In addition, the amplitude of the inward current triggered by application of glutamate (30 $\mu \mathrm{M}, 30 \mathrm{~s})$ was more important for RD cells $(-1.46 \pm 0.2 \mathrm{nA})$ than for biphasic cells $(-0.51 \pm 0.09 \mathrm{nA} p<0.005)$ (Fig. $5 B$ ) but was weaker than the current associated with an ID response $(-4.3 \pm 1.4$

Figure 3. Determination of the $\mathrm{IC}_{50}$ for glutamate receptor antagonists. $A$, Inhibition of glutamate-induced optical response by DNQX (biphasic response, $\boldsymbol{A}$; RD response, $\boldsymbol{A 2}$ ). $\boldsymbol{B}$, Inhibition of glutamate-induced optical response by MK801 (biphasic response, $B 1 ; R D$ response, $B 2$ ). Note that the maximum value for the biphasic response corresponds to the peak amplitude of the phase-shift increase with the lowest antagonist concentration. In contrast, the maximum value for RD response corresponds to the peak amplitude of the phase-shift decrease observed with the lowest antagonist concentration. For each graph, inserts represent a set of phase shifts obtained after the successive application of antagonist $(0.004,0.4$, and $100 \mu \mathrm{m})$ for the same application of glutamate $\left(30 \mu \mathrm{M}, 30 \mathrm{~s}\right.$, arrowhead) onto the same cell. Fitting the data to the logistic equation yielded an $\mathrm{IC}_{50}$ for DNQX of 120 and $110 \mathrm{~nm}$, respectively, for biphasic and RD responses. The $\mathrm{IC}_{50}$ values for MK801 were 170 and $760 \mathrm{~nm}$, respectively, for biphasic and RD responses. Calibration: 2 min and $1^{\circ}$.

of the ID response. Moreover, the simultaneous recording of membrane current and phase signal showed clearly that the ID response is associated with a robust inward current $(-4.3 \pm 1.4$ $\mathrm{nA} ; n=3$ ) reaching a plateau (Fig. 4C). This type of current has
$\mathrm{nA} ; n=3)($ Fig. $4 B)$. Finally, associated with the biphasic and RD responses, patched cells developed a sustained synaptic activity composed by sEPSCs and, occasionally, by sIPSCs (Fig. 5B). However, analysis of sEPSCs (only single-peak events) showed 
that, in the absence of a significant difference in the frequency and amplitude of sEPSCs (Table 1), their kinetics were significantly slower for RD cells (rise time, $4.67 \pm 0.58 \mathrm{~ms}$; decay time, $14.79 \pm 2.7$ $\mathrm{ms}$ ) than for biphasic cells (rise time, $3.00 \pm 0.24, p<0.05$; decay time, $7.93 \pm$ $0.56 p<0.05$ ) (Table 1), suggesting a larger NMDA component in the sEPSCs of RD cells. This is consistent with the notion that the kinetics of these receptors is slower than the non-NMDA receptors (Collingridge and Lester, 1989). Thus, these results indicate that the optical responses are related to the level of activation of NMDA receptors, which themselves depend on the level of neuronal excitability.

Last, if the optical responses are related to the level of neuronal activity, we should be able to modify the phase signal by modulating the level of excitability via the inhibition or activation of inhibitory input. Accordingly, we have performed two types of experiments by using either GABA or the specific $\mathrm{GABA}_{\mathrm{A}}$ receptor antagonist picrotoxin, depending on the previous optical response developed by cells. Thus, in the presence of GABA (3 $\mu \mathrm{M}$ ), an $\mathrm{RD}$ response (assumed to reflect a strong NMDA activity) is changed to a biphasic one $(n=6)$ (Fig. $5 C)$. In the same conditions, control experiments showed clearly that, at resting potential, GABA (3 $\mu \mathrm{M})$ hyperpolarized the membrane potential $(\Delta \mathrm{Vm}=-6.6 \pm$ $1.5 \mathrm{mV} ; n=7$ ) (Fig. 5C). Conversely, in the presence of picrotoxin $(30 \mu \mathrm{M})$, the biphasic responses (assumed to reflect a lowlevel NMDA) triggered by application of glutamate are converted to ID responses (assumed to reflect the death-cell process; $n=6$ ) (Fig. 5D), consistent with the fact that picrotoxin triggers hyperexcitability resulting in epileptiform activity (Straub et al., 1994), leading to the cell death (Muir et al., 1996). Furthermore, our electrical recordings clearly demonstrated that neurons treated with picrotoxin developed an "epileptic activity" consisting of action potentials bursting superimposed on plateau potentials reaching amplitudes up to $30 \mathrm{mV}$ (Fig. 5D).

\section{The optical biphasic and RD responses are not related to cell phenotype}

In view of the presence of two distinct types of individual optical responses, biphasic and $\mathrm{RD}$, we considered the possibility that they could reflect the responses of two distinct cell types present in the cultures, notably glutamate- versus GABA-containing neurons. Indeed, a difference in sensitivity for NMDA has been shown to be associated with cell phenotype in cultures of cortical neurons (Tecoma and Choi, 1989). To address this issue, we injected single neurons with biocytin and visualized them with Steptavidin-Texas Red. As seen in Figure 6, the dendritic arbor of these neurons could extend several hundreds of micrometers from the cell soma. Immunostaining with an antibody against GAD, the enzyme of GABA synthesis, revealed a marked positivity for GAD in the soma of a minority of injected neurons (23\%) (Fig. 6B), which is in agreement with the literature (Kato-Negishi et al., 2004). Furthermore, GAD-

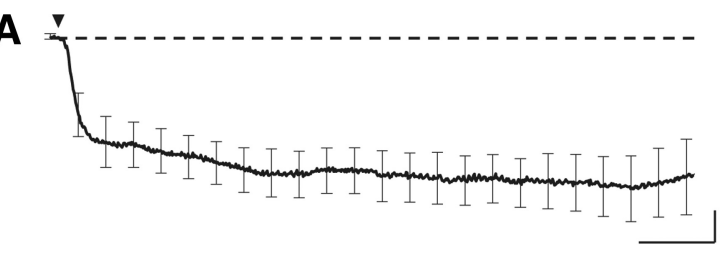

C
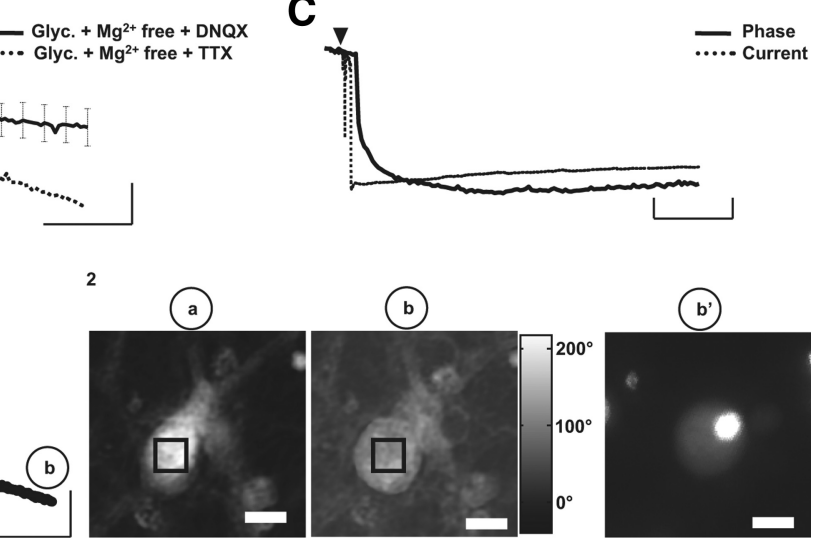

4. ID responses as the optical signal of cell death. $A$, Averaged trace of ID response $(n=6)$ triggered by a short 作 reation: 2 min, $5^{\circ}$, and $1 \mathrm{nA}$. D1, Example of an optical trace corresponding to an ID response triggered by

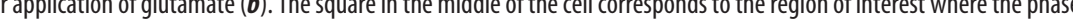
signal is recorded in $\mathbf{C}$. In $\boldsymbol{b}^{\prime}$, the image shows the same neuron as in $\boldsymbol{b}$ after propidium iodide staining. Note that the strong labeling essentially localized to the nucleus of the cell. Scale bars, $10 \mu \mathrm{m}$.

negative cells expressed on their surface GAD-positive puncta suggested that they received GABAergic inputs (Fig. 6A). However, no correlation was found between the reactivity to GAD and either type of optical response, the proportion of $\mathrm{GAD}^{+} / \mathrm{GAD}^{-}$being the same for biphasic and $\mathrm{RD}$ responses (respectively, 3/10 and 2/9) after application of glutamate (Fig. 6B).

\section{Contribution of GABAergic neurotransmission to glutamate-induced optical responses}

As illustrated by experiments presented in Figure 5, $C$ and $D$, modulation of GABA activity (activation or blockade of $\mathrm{GABA}_{\mathrm{A}}$ receptors) affected the shape of the glutamate-induced optical response. This effect seemed indirectly mediated through the control of neuronal excitability (Fig. $5 C, D$ ). Nevertheless, we considered the possibility of a GABAergic contribution (intrinsically associated with a chloride conductance activation) in the genesis of glutamate-induced optical response. This question could be relevant because we obtained electrophysiological (recording of IPSPs) (Fig. 5D) and morphological (GAD-positive cells and GAD-positive puncta around cell bodies) (Fig. 6A) data showing direct GABA inputs in cultured neurons. Furthermore, we can assume that under our experimental conditions, which involve bath application of glutamate, global stimulation of the neuronal network would result in a glutamate-induced GABA release.

To determine such a potential contribution of GABAactivated chloride currents in the production of the optical signal, we have monitored putative changes of the phase-shift signal during exogenous application of GABA (30 $\mu \mathrm{M}, 30 \mathrm{~s})$ on depo- 

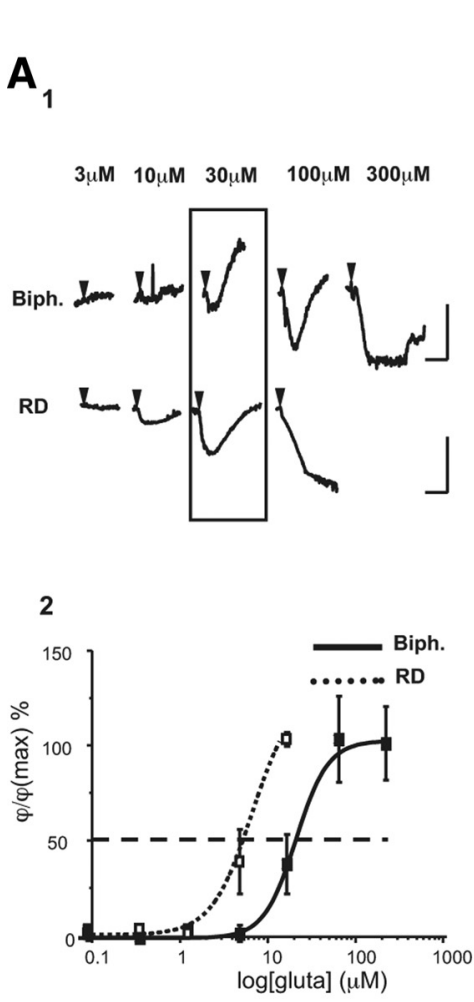

B<smiles>[Mg]</smiles>

Biph.

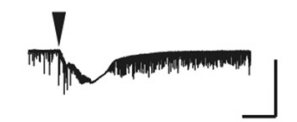

2

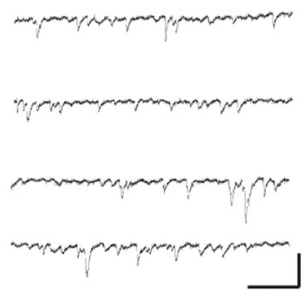

3

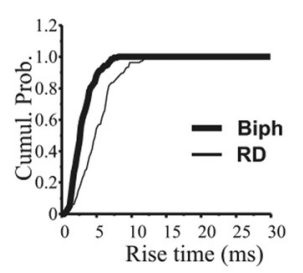

C
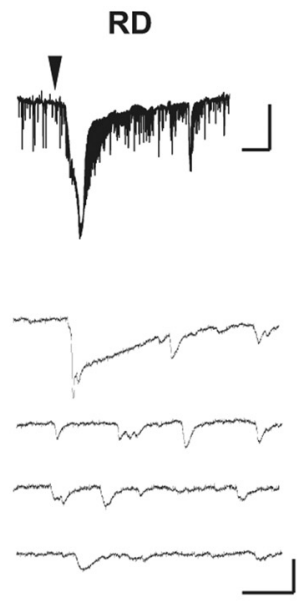

D

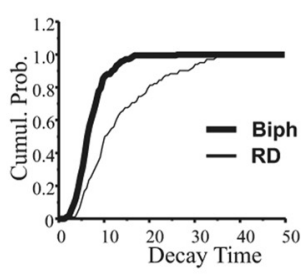

1

2
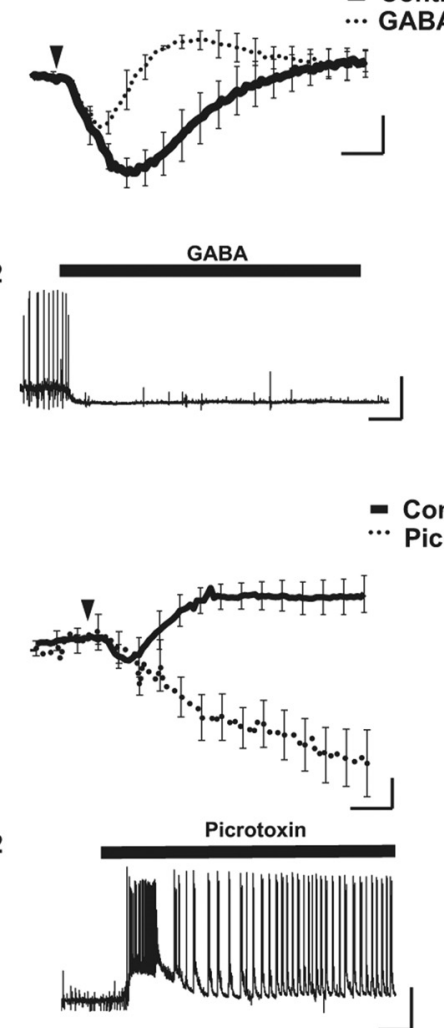

Figure 5. The biphasic response is associated with a lower level of neuronal activity than the RD response. $\mathbf{A 1}$, Examples of two sets of phase shifts obtained after the successive application of glutamate (from $300 \mathrm{~nm} \mathrm{to} 300 \mu \mathrm{m}, 30 \mathrm{~s}$; arrowhead) to the same cell. Classification of the response is determined for a standard application of glutamate ( $30 \mu \mathrm{m}, 30 \mathrm{~s}$ ), as indicated by the rectangle. Calibration: $1 \mathrm{~min}$ and $10^{\circ}$. A2, The graph reports the amplitude of the initial decrease of the phase shift for cells displaying either a biphasic $(n=5)$ or RD $(n=4)$ response. The curve shown is the best fit of the data to the logistic equation described in the Materials and Methods section. Fitting the data to the logistic equation yielded an $\mathrm{EC}_{50}$ of 15 and $45 \mu \mathrm{M}$, respectively, for cells with RD and biphasic responses. B1, Traces of current triggered by glutamate (30 $\mu \mathrm{m}, 30 \mathrm{~s}$, arrowhead) and recorded from cells displaying a biphasic (left) or a RD (right) response. Note that the RD optical response is associated with a stronger inward current than with the biphasic one. Calibration: $0.5 \mathrm{nA}$ and $30 \mathrm{~s}$. B2, Expanded traces of EPSCs from electrical recording shown in $\boldsymbol{B}$, before application of glutamate. Calibration: $100 \mathrm{pA}$ and $50 \mathrm{~ms}$. B3, Cumulative probability plots comparing rise time (bin:0.2 ms; left) and decay time (bin: $0.5 \mathrm{~ms}$; right) for the cells shown in B1 and B2. Kinetics were significantly longer for RD cells as well for rise time (KS two-sample test, $p<0.001$ ) as for decay time (KS two-sample test, $p<0.001$ ). $C 1$, In the presence of GABA ( $3 \mu \mathrm{M}$ ), the RD response obtained in control conditions (Cont.; thick line) became a biphasic response (dotted line) after an application of glutamate (30 $\mu \mathrm{M}, 30 \mathrm{~s}$, arrowhead) $(n=5)$. Scale bars correspond to 1 min and $5^{\circ}$. $\mathbf{C}$, Example of electrophysiological recording from a neuron (displaying tonic activity of action potentials) in culture. Application of GABA (30 $\mu$ m) hyperpolarized the membrane potential with disappearance of action potentials. Calibration: $1 \mathrm{~min}$ and $20 \mathrm{mV}$. D1, Conversely, in the presence of picrotoxin ( $30 \mu \mathrm{m}$ ), the biphasic response obtained in control conditions (Cont.; thick line) became an ID response (dotted line) after an application of glutamate $\left(30 \mu \mathrm{m}, 30 \mathrm{~s}\right.$, arrowhead) $(n=6)$. Calibration: 1 min and $5^{\circ}$. D2, Example of electrophysiological recording from neuron in culture displaying synaptic activity (EPSPs and IPSPs) but no action potential. After application of picrotoxin ( $30 \mu \mathrm{M})$, the electrical activity is characterized by the appearance of an epileptic form of activity (action potentials superimposed on plateau potential). Calibration: 1 min and $20 \mathrm{mV}$.

Table 1. Comparison between sEPSC recorded in biphasic and RD cells

\begin{tabular}{lrr}
\hline & \multicolumn{1}{c}{$s$ EPSC biphasic } & \multicolumn{1}{c}{$s$ EPSC RD } \\
\hline Frequency (Hz) & $5.64 \pm 0.63$ & $3.15 \pm 1.07(p=0.10)$ \\
Amplitude (pA) & $40.24 \pm 6.22$ & $55.46 \pm 7.71(p=0.13)$ \\
Rise time (ms) & $3.00 \pm 0.24$ & $4.67 \pm 0.58(p=0.02)$ \\
Decay time (ms) & $7.93 \pm 0.56$ & $14.79 \pm 2.75(p=0.03)$ \\
\hline
\end{tabular}

Values are given as means \pm SE. Statistical analysis was performed by using an unpaired $t$ test to compare sEPSC biphasic $(n=20)$ and SEPSC RD $(n=10)$.

larized neurons $(0 \mathrm{mV})$ to mimic the depolarizing effect of glutamate. In these conditions, GABA triggered a reversible decrease of phase signal $\left(-6.3 \pm 2.46^{\circ} ; n=6\right)$, with this optical response disappearing when the membrane potential was set at $-60 \mathrm{mV}$ $\left(0.29 \pm 0.51^{\circ}\right)($ Fig. $7 A)$. Moreover, in neurons at resting potential $(-55 \pm 3 \mathrm{mV} ; n=6)$, perfusion of GABA $(3 \mu \mathrm{M})$ resulting in weak hyperpolarization of the membrane potential $(<10 \mathrm{mV})$ (Figs. $5 C, 7 B$ ) did not trigger a detectable optical signal (Fig. $7 B$ ), confirming the role of depolarization in producing an optical signal associated with GABA stimulation. Finally, a short application of GABA (30 $\mu \mathrm{M}, 30 \mathrm{~s})$ was unable to produce an optical response $\left(-0.25 \pm 0.56^{\circ} ; n=7\right)$ on unpatched cells (Fig. $\left.7 A\right)$, even at high concentrations (up to $100 \mu \mathrm{M} ; n=8$ ) (Fig. $7 C$ ).

The second component of the biphasic and $\mathrm{RD}$ response is related to the activation of the cotransporters KCC2 and/or NKCC1

As described in the Introduction, it is now well established that, following depolarization, the two cotransporters NKCC1 and KCC2 operate to restore ionic homeostasis. Accordingly, in the presence of a nonspecific blocker of both cotransporters, furosemide (100 $\mu \mathrm{M} ; 30-40 \mathrm{~min})$, only the small initial decrease of the phase signal in biphasic response persisted (Fig. $8 \mathrm{~A}$ ), with the increase of optical signal being significantly inhibited (from $4.43 \pm 1.08^{\circ}$ to $0.54 \pm 0.83^{\circ} ; p<0.005, n=6$ ) (Fig. $8 A$ ) with an $\mathrm{IC}_{50}$ of $580 \mathrm{~nm}(n=6)$ (Fig. $\left.8 A\right)$. As furosemide is a broadspectrum blocker, we also used a specific inhibitor for NKCC1, bumetanide ( $20 \mu \mathrm{M}, 30-40 \mathrm{~min})$. At this concentration, the biphasic responses were not altered $\left(3.44 \pm 0.55^{\circ}\right.$ vs $3.16 \pm 0.46^{\circ}$; $p=0.9 ; n=6$ ) (Fig. $8 B$ ), suggesting that the blockade obtained with furosemide is due essentially to KCC2 activity. This result is 
in good agreement with the fact that the activation of the KCC2 corresponds to an extrusion of $\mathrm{K}^{+}, \mathrm{Cl}^{-}$, and water (MacAulay et al., 2004); this directionality of the ionic fluxes should indeed result in an increase in the phase signal when this cotransporter is activated.

For RD responses, furosemide (100 $\mu \mathrm{M} ; 30-40 \mathrm{~min}$ ) strongly and significantly diminished the amplitude of the optical response (from $-7.98 \pm 2.28^{\circ}$ to $-1.51 \pm 0.26^{\circ}, p<0.05, n=6$ ) (Fig. $8 A$ ), with an $\mathrm{IC}_{50}$ to $550 \mathrm{nM}$. Moreover, contrary to the biphasic response, bumetanide (20 $\mu \mathrm{M}, 30-40 \mathrm{~min})$ significantly reduced the amplitude of the $\mathrm{RD}$ (from $-7.03 \pm 2.26^{\circ}$ to $-3.88 \pm 1.15^{\circ}, p<$ 0.005, $n=14 ; \mathrm{IC}_{50}=55 \mathrm{~nm}$ ) (Fig. $8 B$ ). This result indicates that the two cotransporters are involved in the genesis of the $\mathrm{RD}$ response. Knowing that the NKCC1 cotransports $\mathrm{K}^{+}, \mathrm{Na}^{+}$, and $\mathrm{Cl}^{-}$in a respective ratio of 1:1:2 and triggers water inflow (Russell, 2000; MacAulay et al., 2004), we can legitimately postulate that the combined activation of glutamate receptors and the NKCC1 caused the decrease in the signal phase, while KCC2 mediated a delayed return to initial values of the signal phase.

\section{Discussion}

Using the properties of water diffusion through the plasma membrane, we show in this study that DHM affords the noninvasive determination of the integrity status of cultured cortical neurons as revealed by the shape and amplitude of optical responses obtained after a short application of glutamate. Indeed, depending on the level of activation, glutamate may play a physiological or pathological role (Meldrum, 2000). Thus, the level of glutamate receptor activation is translated, at the cellular level, in a variable net inflow of cations $\mathrm{Na}^{+}$and/or $\mathrm{Ca}^{2+}$, depending on the glutamate receptors involved). We know that such ionic movements are accompanied by an entry of water to locally maintain the ionic homeostasis in the cell (Olney et al., 1986). Under our experimental conditions, the phase optical signals detected by DHM reflected the activation of glutamate receptors. Indeed, a short application of glutamate triggered an early decrease in the phase signal, corresponding to a passive entry of water (accompanying the inflow of $\mathrm{Na}^{+}$and $\mathrm{Ca}^{2+}$ ). These initial decreases in optical signal have small amplitudes for biphasic responses or a larger one for RD and ID responses. The pharmacology of the glutamate-evoked optical response strongly suggests that the amplitude of the initial decrease in the phase signal is mainly linked to the activation of NMDA receptors, since activation solely of the non-NMDA receptors generates only modest residual biphasic responses (i.e., biphasic-like responses). In contrast, strong activation of NMDA receptors is at the origin of the RD and ID responses, a condition when the NMDA receptor activation is excitotoxic. This correlation between the level of NMDA receptor activation and the amplitude of the initial decrease in phase signal is reinforced by electrophysiological data, since, in addition to a resting potential more depolarized, the cells showing an RD response exhibited stronger glutamate-evoked currents resulting in a larger NMDA component of the sEPSC than for biphasic cells. Finally, it is well established that excessive activation of NMDA receptor causes a marked cell swelling associated with an entry of
Biocytin
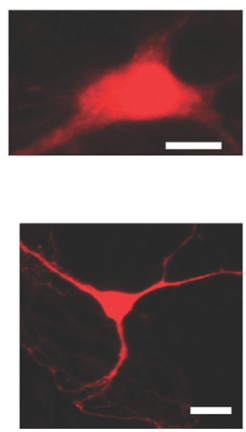

GAD
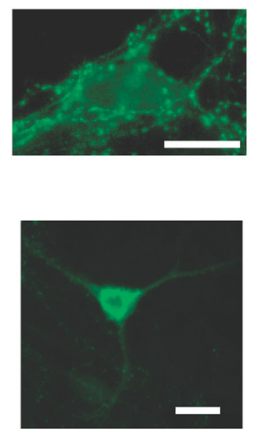

Merged
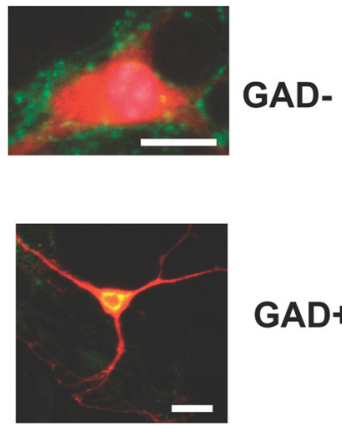

GAD+
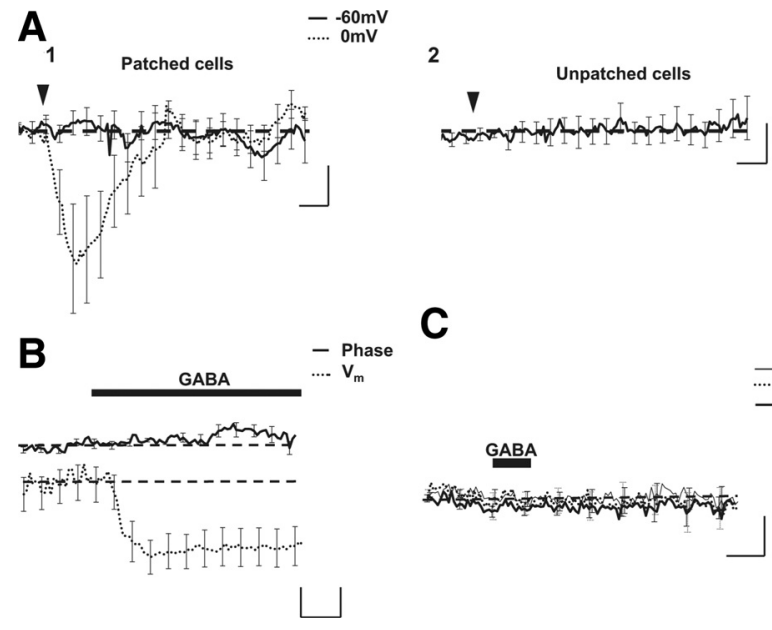

Figure 7. GABA triggers an optical signal only when membrane potential is depolarized. $\mathbf{A 1}$, Averaged optical traces after application of GABA $(30 \mu \mathrm{m}, 30 \mathrm{~s})$ on patched neurons $(n=6)$ at $-60 \mathrm{mV}$ (full line) or at $0 \mathrm{mV}$ (dotted line). Note that GABA triggers an optical response only when the membrane potential was depolarized. $\boldsymbol{A} 2$, Same application of GABA on nonpatched neurons $(n=7)$ from the same culture. In these conditions, GABA did not elicit an optical signal.

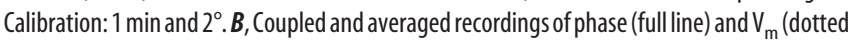
line) obtained after application of GABA ( $30 \mu \mathrm{m}, 30 \mathrm{~s}$, arrowhead) show a hyperpolarization of membrane potential triggered by GABA, which is not associated with detectable phase-shift changes $(n=7)$. Calibration: $1 \mathrm{~min}, 2^{\circ}$, and $2 \mathrm{mV}$. C, Averaged optical traces obtained after the successive application of GABA (1-100 $\mu \mathrm{m}, 30$ s; arrowhead) to the same unpatched cells ( $n=$ 8). No optical response was observed in these conditions.

water (Collingridge and Lester, 1989) which may lead to cell death (Andrew et al., 1996). Thus, ID responses corresponding to the strongest decrease in the phase signal were triggered only if NMDA receptors were activated and were associated with a persistent depolarizing current already described in the literature as being associated with pathological processes leading to cell death (Limbrick et al., 2001).

In addition to the pure glutamate component, a glutamateinduced GABA release appears to also participate in the phase shift observed during glutamate application. This GABA component is linked to the inflow of water accompanying the inflow of $\mathrm{Cl}^{-}$and are proportional to the level of depolarization of the membrane potential. However, experiments in which exogenous GABA was applied to the cultures indicate that the reversible 

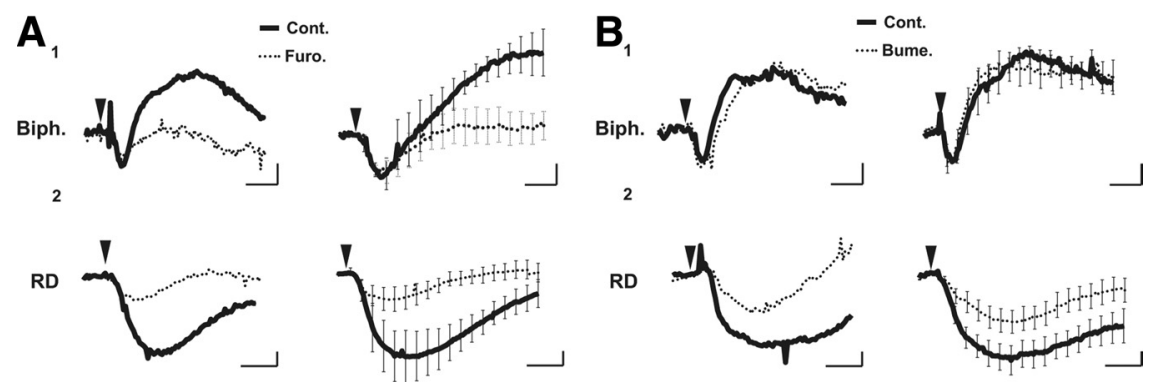

RD
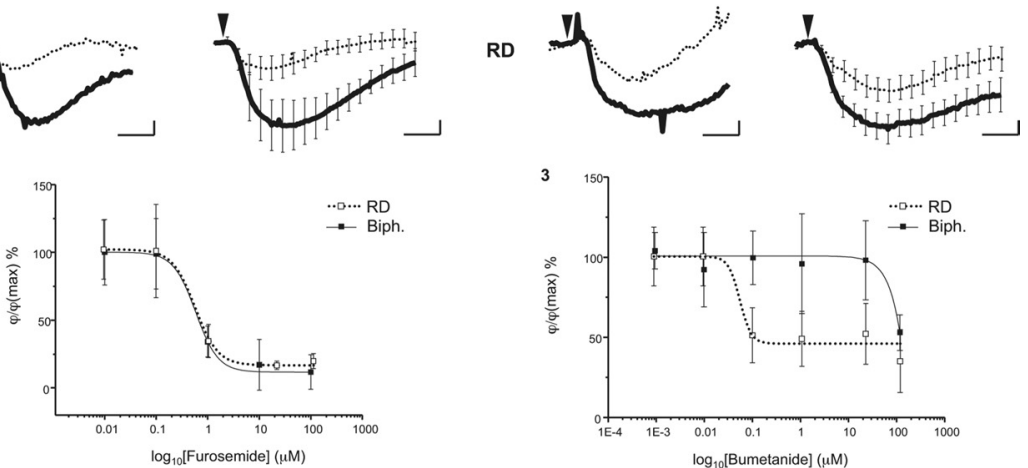

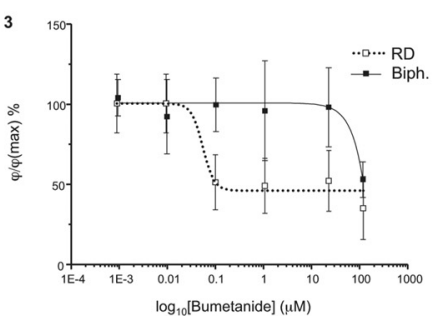

Figure 8. KCC2 and NCKK1 are involved in the production of biphasic and RD responses. $A 1$, Single (left) and averaged $(n=6$; right) biphasic responses triggered by glutamate ( $30 \mu \mathrm{m}, 30 \mathrm{~s}$, arrowhead) in control condition (full line) and in the presence of furosemide (Furo.; $100 \mu \mathrm{m}$, dotted line). Note that only the second part of the optical signal (increase of the phase shift) was blocked by furosemide. $\boldsymbol{A 2}$, Single (left) and averaged ( $n=6$; right) RD response triggered by glutamate ( $30 \mu \mathrm{m}, 30$ s, arrowhead) in control condition (full line) and in the presence furosemide (dotted line). The amplitude of $\mathrm{RD}$ response is strongly decreased by furosemide. $A 3$ : Concentration-response curves of the inhibitory effect of furosemide on biphasic (full square and full line; $I C_{50}=$ $580 \mathrm{~nm}$ ) and RD (empty square and dotted line; $K_{50}=550 \mathrm{~nm}$ ) responses. $B$ 1, Single (left) and averaged ( $n=6$; right) biphasic responses triggered by glutamate (30 $\mu \mathrm{M}, 30 \mathrm{~s}$, arrowhead) in control condition (full line) and in the presence of bumetanide (Bume.; $20 \mu \mathrm{m}$, dotted line). With this blocker, there were no modifications of the biphasic responses. B2, Single (left) and averaged ( $n=14$; right) RD responses triggered by glutamate ( $30 \mu \mathrm{M}, 30 \mathrm{~s}$, arrowhead) in control condition (Cont.; full line) and in the presence of bumetanide (dotted line). The amplitude of the $R D$ response is decreased by bumetanide to a smaller degree than by furosemide. B3, Concentration-response curves of the inhibitory effect of bumetanide on biphasic (Biph.; full square and full line; $I_{50}$ not determined) and RD (empty square and dotted line; $I_{50}=55 \mathrm{~nm}$ ) responses. Note that an inhibitory effect of bumetanide was observed from $100 \mu \mathrm{m}$ on for the biphasic response, a concentration 2000-fold higher than the $\mathrm{IC}_{50}$ observed for the RD response. Calibration: 2 min and $2^{\circ}$.

decrease in the phase signal triggered during depolarizing conditions was not followed by an optical "overshoot" (Fig. 7A), suggesting that the second part of biphasic response was independent of GABA activity. Moreover, the duration of the pure GABA-induced response was significantly shorter $(220 \pm$ $45 \mathrm{~s} ; n=7 ; p<0.001)$ than a $\mathrm{RD}$ response $(550 \pm 23 \mathrm{~s} ; n=65)$, indicating that, in this type of optical response too, the slow return of phase shift to the initial value was not due to a slow decrease in GABA activity but that it was linked to another mechanism, with slower kinetics. Overall, this set of experiments suggests that endogenous GABA released during activation of the neuronal network by glutamate could partially participate in the initial part of glutamate-induced optical response corresponding to the phase-shift decrease (additional inflow of $\mathrm{Na}^{+}, \mathrm{Ca}^{2+}$ and $\mathrm{Cl}^{-}$) of biphasic and $\mathrm{RD}$ responses, but most likely not in the second part of these optical signals. Finally, the fact that glutamate triggered almost exclusively ID responses in the presence of picrotoxin indicates that the endogenous GABAergic activity exerted an inhibitory effect resulting in protection against excitotoxicity induced by exogenous glutamate application, which is consistent with previous reports (Muir et al., 1996).

In summary, DHM allows distinguishing between two glutamate-mediated responses, as follows: first, the ID, which will eventually lead to cell death (Pavillon et al., 2010); and second, two distinct responses, biphasic and RD, which depend on the resting membrane potential of the neuron.

In addition, DHM is the first imaging technique offering the possibility to dynamically and directly monitor the activities of the two neuronal cotransporters, NKCC1 and KCC2 in situ. This is based on the fact that NKCC1 and KCC2 cotransport ions and water (MacAulay et al., 2004), an underlying phenomenon of the optical signals recorded by DHM. The activation of KCC2 results in the extrusion of ions and water, which produces an increase in the phase signal detectable by DHM; in contrast, activation of NKCC1 results in a decrease in the phase signal associated with an entry of ions and water. Results obtained with cotransporter blockers confirmed this working hypothesis since the inhibition of KCC2 by furosemide inhibits the second part (the rise) of the two types of phase signals, while specific NKCC1 inhibition reduces the amplitude of the decrease of phase signal only during an $\mathrm{RD}$ response. These results are consistent with the pharmacology of glutamate receptors in the genesis of optical signals. Indeed, NKCC1 and $\mathrm{KCC} 2$ are regulated in opposing ways by intracellular $\mathrm{Ca}^{2+}$. Thus, the activity of NKCC1 is stimulated by increased intracellular $\mathrm{Ca}^{2+}$ (Schomberg et al., 2003), while that of KCC2 is inhibited (Fiumelli et al., 2005). Since NMDA receptor activity results in $\mathrm{Ca}^{2+}$ entry into neurons, it is not surprising to observe that the activation of NMDA receptors produces optical signals consistent with NKCC1 stimulation and KCC2 inhibition. This dual action of NMDA receptor-dependent activation results in the strong decrease in the phase signal observed in the $\mathrm{RD}$ response and in the apparent delayed activation of KCC2. It is important to note that an excessive activation of NKCC1 contributes to the process of excitotoxicity (Beck et al., 2003). Interestingly, the ID response detected with DHM and associated with the subsequent cell-death process, is consistent with this view because such optical responses were never recorded after blockade of NKCC1 by bumetanide (or furosemide).

NKCC1 and KCC2 cotransporters are involved in the homeostasis of $\mathrm{Cl}^{-}$ions (Payne et al., 2003) since KCC2 is activated by the intracellular increase of $\mathrm{Cl}^{-}$while NKCC1 is, in contrast, inhibited (Lytle and McManus, 2002). Thus, inhibition of GABAergic activity with picrotoxin will result in a decreased entry of $\mathrm{Cl}^{-}$into the target cell. This should result in an increase in the activity of NKCC1 and inhibition of activity of KCC2 consistent with the marked decrease in the phase signal. In contrast, in the presence of $\mathrm{GABA} \mathrm{Cl}^{-}$inflow is stimulated, resulting in decreased activity and membrane hyperpolarization of target neurons, thus preventing NMDA receptor activity and the associated $\mathrm{Ca}^{2+}$ entry. The resulting inhibition of NKCC1 and activation of KCC2 are likely to be the basis of the biphasic response following glutamate application. Concerning this last type of optical response, we have observed that, on certain occasions, the rise in the phase shift was not preceded by a small initial decrease in the biphasic response, as seen in Figure 2. One possible explanation could be an early activation of KCC2 that started immediately after receptor activation. This early activation of KCC2 would result in the exit of ions and water counteracting the ionic and water inflow linked to opening of glutamate (and GABA) recep- 
tors, hence resulting in the absence of a small initial decrease of phase shift.

Finally, knowing that cotransporter blockers disturb the regulation of chloride homeostasis and, by consequence, the efficiency of GABAergic activity, one could suggest that the effects of furosemide (and/or bumetanide) on glutamate-induced optical responses are a direct consequence of GABAergic activity and not of a direct blockade of cotransporter. However, the shape and kinetics of pure GABA-induced optical responses strongly suggested that the GABA optical component was essentially involved in the first part of the biphasic or RD responses (i.e., during glutamate depolarization) rather than in the blockade of cotransporter activity concerned the second part of these same optical signals (i.e., after the end of glutamate depolarization). Thus, it is very unlikely that the optical effects of cotransporter blockade are associated with a modulation of GABAergic activity.

In conclusion, we show here that DHM can detect in a dynamic and noninvasive manner the activity of membrane proteins that mediate transmembrane ion and water transport. More precisely, in this article we have examined the signals mediated by two neurotransmitters (glutamate and GABA) on their receptors, as well as the activity of two cotransporters, NCKK1 and KCC2. The analysis with appropriate pharmacological tools of the effect of agonists (e.g., glutamate and GABA) or antagonists (e.g., glutamate-receptor antagonists, picrotoxin, furosemide and bumetamide) associated with changes in the DHM signal provides a novel approach for the study of the functional properties of the target membrane proteins. This imaging technique could also be applied to other proteins involved in water transport and cell volume regulation.

\section{References}

Andrew RD, MacVicar BA (1994) Imaging cell volume changes and neuronal excitation in the hippocampal slice. Neuroscience 62:371-383.

Andrew RD, Adams JR, Polischuk TM (1996) Imaging NMDA- and kainate-induced intrinsic optical signals from the hippocampal slice. J Neurophysiol 76:2707-2717.

Arundine M, Tymianski M (2004) Molecular mechanisms of glutamatedependent neurodegeneration in ischemia and traumatic brain injury. Cell Mol Life Sci 61:657-668.

Barer R (1953) Determination of dry mass, thickness, solid and water concentration in living cells. Nature 172:1097-1098.

Beck J, Lenart B, Kintner DB, Sun D (2003) Na-K-Cl cotransporter contributes to glutamate-mediated excitotoxicity. J Neurosci 23:5061-5068.

Brewer LD, Thibault O, Staton J, Thibault V, Rogers JT, Garcia-Ramos G, Kraner S, Landfield PW, Porter NM (2007) Increased vulnerability of hippocampal neurons with age in culture: temporal association with increases in NMDA receptor current, NR2A subunit expression and recruitment of L-type calcium channels. Brain Res 1151:20-31.

Collingridge GL, Lester RA (1989) Excitatory amino acid receptors in the vertebrate central nervous system. Pharmacol Rev 41:143-210.

Fiumelli H, Cancedda L, Poo MM (2005) Modulation of GABAergic transmission by activity via postsynaptic $\mathrm{Ca} 2+$-dependent regulation of $\mathrm{KCC} 2$ function. Neuron 48:773-786.

Grinvald A, Lieke E, Frostig RD, Gilbert CD, Wiesel TN (1986) Functional architecture of cortex revealed by optical imaging of intrinsic signals. Nature 324:361-364.

Kato-Negishi M, Muramoto K, Kawahara M, Kuroda Y, Ichikawa M (2004)
Developmental changes of GABAergic synapses formed between primary cultured cortical neurons. Brain Res Dev Brain Res 152:99-108.

Le Bihan D (2007) The 'wet mind': water and functional neuroimaging. Phys Med Biol 52:R57-R90.

Lecoeur H (2002) Nuclear apoptosis detection by flow cytometry: influence of endogenous endonucleases. Exp Cell Res 277:1-14.

Limbrick DD Jr, Pal S, DeLorenzo RJ (2001) Hippocampal neurons exhibit both persistent $\mathrm{Ca} 2+$ influx and impairment of $\mathrm{Ca} 2+$ sequestration/ extrusion mechanisms following excitotoxic glutamate exposure. Brain Res 894:56-67.

Lytle C, McManus T (2002) Coordinate modulation of Na-K-2Cl cotransport and $\mathrm{K}-\mathrm{Cl}$ cotransport by cell volume and chloride. Am J Physiol Cell Physiol 283:C1422-C1431.

MacAulay N, Hamann S, Zeuthen T (2004) Water transport in the brain: role of cotransporters. Neuroscience 129:1031-1044.

Marquet P, Rappaz B, Magistretti PJ, Cuche E, Emery Y, Colomb T, Depeursinge C (2005) Digital holographic microscopy: a noninvasive contrast imaging technique allowing quantitative visualization of living cells with subwavelength axial accuracy. Optics Letters 30:468-470.

Meldrum BS (2000) Glutamate as a neurotransmitter in the brain: review of physiology and pathology. J Nutr 130:1007S-1015S.

Mody I, MacDonald JF (1995) NMDA receptor-dependent excitotoxicity: the role of intracellular Ca2 + release. Trends Pharmacol Sci 16:356-359.

Muir JK, Lobner D, Monyer H, Choi DW (1996) GABAA receptor activation attenuates excitotoxicity but exacerbates oxygen-glucose deprivationinduced neuronal injury in vitro. J Cereb Blood Flow Metab 16:1211-1218.

Nicoletti I, Migliorati G, Pagliacci MC, Grignani F, Riccardi C (1991) A rapid and simple method for measuring thymocyte apoptosis by propidium iodide staining and flow cytometry. J Immunol Methods 139:271-279.

Olney JW, Price MT, Samson L, Labruyere J (1986) The role of specific ions in glutamate neurotoxicity. Neurosci Lett 65:65-71.

Pavillon N, Benke A, Boss D, Moratal C, Kühn J, Jourdain P, Depeursinge C, Magistretti PJ, Marquet P (2010) Cell morphology and intracellular ionic homeostasis explored with a multimodal approach combining epifluorescence and digital holographic microscopy. J Biophotonics 3:432-436.

Payne JA, Rivera C, Voipio J, Kaila K (2003) Cation-chloride cotransporters in neuronal communication, development and trauma. Trends Neurosci 26:199-206.

Rappaz B, Marquet P, Cuche E, Emery Y, Depeursinge C, Magistretti P (2005) Measurement of the integral refractive index and dynamic cell morphometry of living cells with digital holographic microscopy. Optics Express 13:9361-9373.

Rappaz B, Barbul A, Emery Y, Korenstein R, Depeursinge C, Magistretti PJ, Marquet P (2008) Comparative study of human erythrocytes by digital holographic microscopy, confocal microscopy, and impedance volume analyzer. Cytometry A 73:895-903.

Rothman SM (1985) The neurotoxicity of excitatory amino acids is produced by passive chloride influx. J Neurosci 5:1483-1489.

Russell JM (2000) Sodium-potassium-chloride cotransport. Physiol Rev 80:211-276.

Schomberg SL, Bauer J, Kintner DB, Su G, Flemmer A, Forbush B, Sun D (2003) Cross talk between the GABA(A) receptor and the Na-K-Cl cotransporter is mediated by intracellular Cl. J Neurophysiol 89:159-167.

Schwartzkroin PA, Baraban SC, Hochman DW (1998) Osmolarity, ionic flux, and changes in brain excitability. Epilepsy Res 32:275-285.

Straub H, Köhling R, Speckmann EJ (1994) Picrotoxin-induced epileptic activity in hippocampal and neocortical slices (guinea pig): suppression by organic calcium channel blockers. Brain Res 658:119-126.

Tecoma ES, Choi DW (1989) GABAergic neocortical neurons are resistant to NMDA receptor-mediated injury. Neurology 39:676-682. 